\title{
Non-invasive brain stimulation enhances the effects of melodic intonation therapy
}

\author{
Bradley W. Vines ${ }^{1,2}$, Andrea C. Norton ${ }^{1}$ and Gottfried Schlaug ${ }^{1}$ * \\ Department of Neurology, Beth Israel Deaconess Medical Center, Harvard Medical School, Boston, MA, USA \\ 2 Department of Psychiatry, Institute of Mental Health, University of British Columbia, Vancouver, BC, Canada
}

Edited by:

Lutz Jäncke, University of Zurich,

Switzerland

Reviewed by:

Lutz Jäncke, University of Zurich, Switzerland

Eckart Altenmüller, University of

Music and Drama Hannover, Germany

*Correspondence:

Gottfried Schlaug, Music

Neuroimaging, and Stroke Recovery Laboratories, Department of

Neurology, Beth Israel Deaconess

Medical Center, Harvard Medical

School, 330 Brookline Avenue, Palmer

127, Boston, MA 02215, USA

e-mail: gschlaug@bidmc.harvard.edu
Research has suggested that a fronto-temporal network in the right hemisphere may be responsible for mediating melodic intonation therapy's (MIT) positive effects on speech recovery. We investigated the potential for a non-invasive brain stimulation technique, transcranial direct current stimulation (tDCS), to augment the benefits of MIT in patients with non-fluent aphasia by modulating neural activity in the brain during treatment with MIT. The polarity of the current applied to the scalp determines the effects of tDCS on the underlying tissue: anodal-tDCS increases excitability, whereas cathodal tDCS decreases excitability. We applied anodal-tDCS to the posterior inferior frontal gyrus of the right hemisphere, an area that has been shown both to contribute to singing through the mapping of sounds to articulatory actions and to serve as a key region in the process of recovery from aphasia, particularly in patients with large left hemisphere lesions. The stimulation was applied while patients were treated with MIT by a trained therapist. Six patients with moderate to severe non-fluent aphasia underwent three consecutive days of anodal-tDCS + MIT, and an equivalent series of sham-tDCS + MIT. The two treatment series were separated by 1 week, and the order in which the treatments were administered was randomized. Compared to the effects of sham-tDCS + MIT, anodal-tDCS + MIT led to significant improvements in fluency of speech. These results support the hypothesis that, as the brain seeks to reorganize and compensate for damage to left hemisphere language centers, combining anodal-tDCS with MIT may further recovery from post-stroke aphasia by enhancing activity in a right hemisphere sensorimotor network for articulation.

Keywords: melodic intonation therapy, transcranial direct current stimulation, tDCS, Broca's aphasia, stroke, neurorehabilitation, singing

\section{INTRODUCTION}

Of the more than 750,000 strokes that occur every year in the US, approximately $20 \%$ of the survivors suffer from some form of aphasia, an impairment in the ability to express and/or understand speech and language (Kertesz and McCabe, 1977; Mohr et al., 1978; Pedersen et al., 2004; Schlaug et al., 2008a). Though traditional therapies for post-stroke language recovery have been shown to have a beneficial effect (Robey, 1994; Holland et al., 1996), in most cases, patients' recovery is incomplete, particularly when they have large lesions in the left hemisphere. To date, relatively few speech therapy techniques have succeeded in helping these severely impaired patients. However, an intonation-based intervention, melodic intonation therapy (MIT), may offer hope for patients who suffer from severe non-fluent aphasia (Albert et al., 1973; Sparks et al., 1974; Schlaug et al., 2008a,b, 2010). In addition, the synthesis of effective behavioral therapy with complimentary brain stimulation methods to further engage neural centers that are important for recovery and to facilitate neuroplastic changes (Stefan et al., 2000; Adkins-Muir and Jones, 2003; Nair et al., 2008; Celnik et al., 2009; Baker et al., 2010; Lindenberg et al., 2010; Floel et al., 2011; Fridriksson et al., 2011) is a promising frontier for post-stroke neurorehabilitation. Indeed, a combined peripheral and central stimulation approach has recently been shown to enhance synaptic plasticity more than central stimulation alone in an experimental animal study (Fritsch et al., 2010). Thus, combining a speech or intonation-based therapy with noninvasive brain stimulation may be particularly effective as a means for treating non-fluent aphasia.

A stroke affecting the left frontal lobe can cause a form of aphasia known as "non-fluent" or "Broca's" aphasia (Goodglass and Geschwind, 1976; Kertesz et al., 1977; Mohr et al., 1978). Broca’s aphasia is characterized by either a deficit in speech output without significant impairment to comprehension, or an impairment in the ability to organize the elements of speech (e.g., phonemes) into streams of sound that form meaningful utterances. Prior research has suggested that there are two neural pathways with the potential to facilitate recovery from Broca's aphasia. One pathway primarily recruits peri-lesional cortex in the left hemisphere, and may also elicit varying degrees of engagement from the right hemisphere for recovery of language function. This route for recovery is only possible when patients have smaller left hemisphere lesions that have not completely destroyed Broca's region or the surrounding cortex, and thus, may have enough peri-lesional cortex remaining to support language and speech output. Typically, these 
patients have a milder form of aphasia. The other route to recovery relies almost exclusively on the right hemisphere, and may be the only recovery option for patients with large lesions that have destroyed all language-capable structures in the left hemisphere (Mimura et al., 1998; Pizzamiglio et al., 2001; Thiel et al., 2001; Blasi et al., 2002; Winhuisen et al., 2005; Schlaug et al., 2008b). Natural outcome studies in patients with lesions of this kind provide evidence that neural centers in the right hemisphere are capable of supporting vocal-motor output and compensating for damaged "eloquent" areas in the left hemisphere to facilitate language recovery (Mimura et al., 1998; Rosen et al., 2000; Winhuisen et al., 2005; Heiss and Thiel, 2006; Saur et al., 2006). What remains undetermined is how to specifically engage these right-hemispheric vocal-motor centers and enhance their contribution to the recovery process. Based upon the existing literature and our own data, it appears that these right fronto-temporal centers respond well to interventions that emphasize melodic contour and re-map sounds to articulatory actions with the help of a right-hemispheric sensorimotor network that is engaged by rhythmic activities involving the left hand (Schlaug et al., 2008a,b, 2009, 2010; Wan et al., 2010). Although impairments in melodic and temporal music information processing have been associated with acquired lesions in both right and left hemispheres, right hemsiphere-lesioned patients have revealed more of an impairment in melodic contour and meter (Schuppert et al., 2000) suggesting that interventions emphasizing these aspects of an intonation-based therapy might particularly engage right hemisphere structures.

Neuroimaging studies have revealed both unique and overlapping brain networks for speaking and singing (Brown et al., 2004; Ozdemir et al., 2006); although speaking tends to be lateralized to the left hemisphere and singing to the right (Riecker et al., 2000; Jeffries et al., 2003; Sparing et al., 2007), these two behaviors involve some of the same brain areas. In an fMRI study from our group (Ozdemir et al., 2006) we found that singing and speaking shared some bilateral fronto-temporal neural correlates, but singing, or intoned speaking, led to additional activation of the right more than left superior temporal and right more than left central operculum compared to the speaking condition. Thus, singing could be a way to access language-capable regions in the right hemisphere for the purpose of facilitating language recovery (Racette et al., 2006; Schlaug et al., 2008a,b, 2009, 2010; Wan et al., 2010).

One speech therapy that capitalizes on the possible rehabilitative effects of singing in patients with motor aphasias is MIT. This technique was inspired by the common clinical observation that severely aphasic patients can sing the lyrics of songs better than they can speak them (Goldstein, 1942; Gerstman, 1964; Geschwind, 1971; Keith and Aronson, 1975; Kinsella et al., 1988; Hebert et al., 2003). MIT uses a simplified, exaggerated prosody to train patients to intone and tap out high probability words and phrases syllable by syllable. Research has found MIT to be effective in facilitating significant improvements in language production (Albert et al., 1973; Sparks et al., 1974; Laughlin et al., 1979; Bonakdarpour et al., 2000; Wilson et al., 2006; Schlaug et al., 2008a, 2009). MIT's efficacy may be due to its unique ability to engage language-capable brain regions in both hemispheres, but most particulary, in the right hemisphere (Albert et al., 1973; Sparks et al., 1974; Schlaug et al., 2008a). The posterior inferior frontal gyrus (IFG) very likely plays a critical role in the network underlying the rehabilitative effect of MIT (Schlaug et al., 2008a), and it is important to note that this brain region, which includes the right hemisphere homolog of Broca's area, is easily accessible for application of non-invasive brain stimulation.

Transcranial direct current stimulation (tDCS) is a technique that influences excitability in the brain by modulating the spontaneous firing rate of neurons (Priori et al., 1998; Nitsche and Paulus, 2000). Research suggests that the polarity of the current determines the effects of tDCS: anodal-tDCS increases cortical excitability, whereas cathodal tDCS decreases excitability. Activity in sodium and calcium ion channels as well as the efficacy of NMDA receptors may mediate the effects of $\mathrm{tDCS}$ (Liebetanz et al., 2002; Nitsche et al., 2003a). Applying tDCS has been shown to improve cognitive and behavioral performance on tasks involving the stimulated brain area (Nitsche et al., 2003b; Antal et al., 2004; Kincses et al., 2004; Fregni et al., 2005; Boggio et al., 2006a; Vines et al., 2006a). For example, studies have found that applying anodal-tDCS to regions in the left frontal cortex significantly improved fluency in healthy participants (Iyer et al., 2005; Cattaneo et al., 2011). We have also recently shown (Zheng et al., 2011) that tDCS can lead to modality dependent regional blood flow increases in targeted brain regions, and that resting regional blood flow after tDCS is turned off shows longer lasting effects that differ between anodal and cathodal stimulation. These findings are in agreement with the longer-term electrophysiological effects of either increased excitability (anodal) or decreased excitability (cathodal). Researchers have already employed tDCS as a tool to facilitate recovery from dysfunctions caused by a stroke (Schlaug and Renga, 2008; Schlaug et al., 2008c; Baker et al., 2010; Fiori et al., 2010; Lindenberg et al., 2010; Floel et al., 2011; Fridriksson et al., 2011; Schlaug et al., 2011). In the context of speech recovery, at least one study has investigated the potential for tDCS alone to facilitate recovery from non-fluent aphasia (Monti et al., 2008). And there is a precedent for combining tDCS with a behavioral speech therapy with positive results (Baker et al., 2010; Floel et al., 2011; Fridriksson et al., 2011). We sought to combine tDCS with the intonation-based speech therapy, MIT, for two reasons: (1) preliminary imaging results indicate a leading role for the right posterior IFG in the MIT-induced recovery process, and (2) no study has yet tested whether applying anodal stimulation vs sham stimulation in combination with MIT to undamaged right hemisphere structures will lead to an improvement in speech output/fluency for patients with non-fluent aphasia (see also Hamilton et al., 2011 and Schlaug et al., 2011 for more details).

The present study contrasted the effects of two tDCS conditions (anodal and sham) when applied over the right IFG during MIT sessions. We hypothesized that, compared to sham, applying anodal-tDCS concurrently with MIT would enhance neural activity, and synaptic plasticity in the right-hemispheric brain regions that drive the positive effects of MIT on speech production and fluency, and thereby promote a greater degree of recovery.

\section{MATERIALS AND METHODS PARTICIPANTS}

Six patients (all males) with non-fluent aphasia participated in this study. The protocol was approved by the Beth Israel Deaconess 
Medical Center Institutional Review Board and all participants gave written informed consent. All patients were at least 1 year post-onset of their first (and only) ischemic stroke. All patients' strokes affected their left frontal lobe (see combined lesion map of all six patients shown in Figure 1). Lesion volumes ranged from 60 to 218 cc (see Table 1; for more details on lesion calculations see Marchina et al., 2011). Based on our baseline assessments which included the Boston Diagnostic Aphasia Examination (BDAE), our patients were classified as having moderate to severe nonfluent Broca's aphasia with relatively unimpaired comprehension. All six patients had previously been part of a "proof-of-concept" study in our laboratory assessing the behavioral and neural effects of MIT (for more details on preliminary results of this study see Schlaug et al., 2008a,b, 2009, 2010), which they had completed at least 6 months prior to participating in the current protocol of tDCS + MIT. Thus, our patients were familiar with MIT, but had not participated in any study involving MIT for at least 6 months prior to being enrolled into the current study. All patients had a stable baseline prior to enrollment in this study. The ages of the patient participants ranged from 30 to 81 years. Five of the patients were right handed and one was mixed-handed. One of the six participants was bi-lingual (Russian and English); all other participants were native speakers of English.

\section{PROCEDURE}

Participants underwent two series (one series per tDCS condition) of three therapy sessions each. Treatment sessions were administered one per day for three consecutive days. The two series were separated by 1 week. During the treatment sessions, we applied tDCS to the right posterior IFG with the electrode angled toward the temporal lobe. The stimulation period lasted for $20 \mathrm{~min}$ and overlapped with a 20 -min session of MIT administered by a trained therapist. The therapist tailored each MIT session to the skill level of the participant. For one 3-day therapy series, we applied anodal-tDCS, and for the other, sham-tDCS. The ordering of the two stimulation conditions was counterbalanced across participants such that half of them received the sham-tDCS series first, and whether participants started with the sham or anodal stimulation was determined in a randomized fashion. tDCS was applied for $20 \mathrm{~min}$, with the active electrode positioned over the participants' right IFG, and centered approximately $2.5 \mathrm{~cm}$ posterior to $\mathrm{F} 8$ of the 10-20 International EEG system for electrode placement. Neuroimaging studies have confirmed the correspondence between F8 and the right midportion of the IFG (Homan et al., 1987; Okamoto et al., 2004), including our own pilot study using high resolution ( $1 \mathrm{~mm}$ three voxel size) structural MRI $(N=5)$. We chose to position the active electrode slightly posterior to F8 (about $2.5 \mathrm{~cm}$ ) based upon our pilot study investigating the location of the right hemisphere's homolog of Broca's area. A number of TMS and tDCS studies have used the 10-20 EEG system to identify the location of brain structures for stimulation (Kincses et al., 2004; Rogalewski et al., 2004; Fregni et al., 2005; Iyer et al., 2005; Vines et al., 2006a,b, $2008 \mathrm{a}, \mathrm{b})$. Due to the size of the active electrode $\left(\right.$ area $\left.=16.3 \mathrm{~cm}^{2}\right)$,

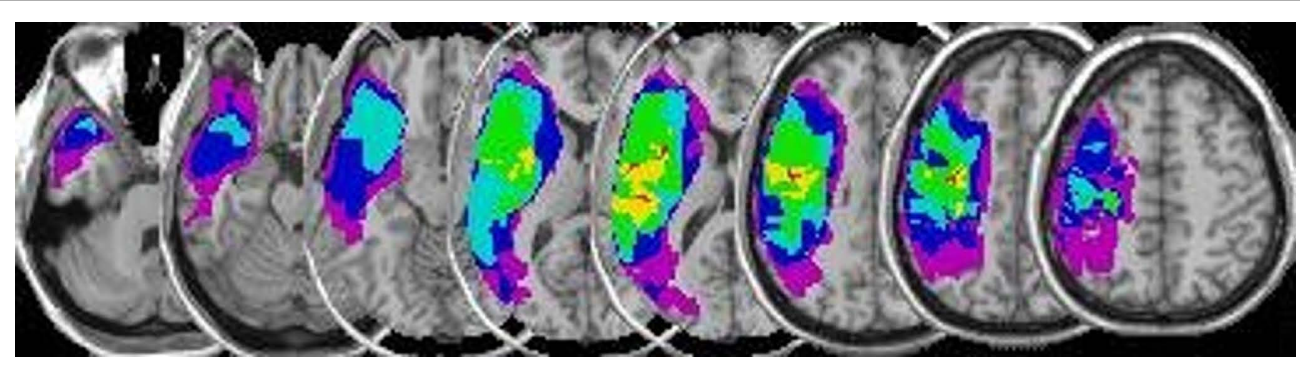

FIGURE 1 | Overlay lesion maps. This figure shows a plot of all lesion maps superimposed onto an individual, spatially standaridzed T1-weighted image (NB: images are displayed in neurological orientation: left side of the image is the left hemisphere). The color scale indicates the extent of overlap among patients with green, yellow, and red indicating overlapping voxels of lesion maps of 4,5 , and 6 patients respectively.

Table 1 | Age at treatment (in years), time post-stroke (in years), total lesion volume (in cubic centimeter), lesion volume overlying a canonical arcuate fasciculus derived from a group of healthy elderly control subjects (AF lesion-load; for more details see Marchina et al., 2011), and fluency changes (in seconds) either after three sessions of anodal-tDCS + MIT or after three sessions of sham-tDCS + MIT.

\begin{tabular}{|c|c|c|c|c|c|}
\hline Age @ Tx & $\begin{array}{l}\text { Times post-stroke } \\
\text { (years) }\end{array}$ & Lesion volume (cc) & AF-lesion-load (cc) & $\begin{array}{l}\text { Fluency, percentage } \\
\Delta \text { in duration (s) }\end{array}$ & $\begin{array}{l}\text { Fluency, percentage } \\
\Delta \text { in duration (s) }\end{array}$ \\
\hline & & & & After anodal-tDCS + MIT & After sham-tDCS + MIT \\
\hline 31.3 & 10.0 & 191.7 & 10.6 & -8.3 & 12.9 \\
\hline 48.8 & 2.5 & 154.8 & 7.6 & -19.8 & 1.1 \\
\hline 80.9 & 1.3 & 60.0 & 3.8 & -12.8 & 5.3 \\
\hline 62.7 & 2.8 & 86.1 & 7.3 & -11.9 & -6.3 \\
\hline
\end{tabular}


the stimulation may have extended into anterior temporal cortex and ventral premotor cortex, which make up the network of fronto-temporal regions that may underlie MIT's therapeutic effect. The reference electrode (area $=30 \mathrm{~cm}^{2}$ ) was positioned over the left supraorbital region. This location for the reference electrode was functionally ineffective in the experimental design (Nitsche et al., 2003b).

A battery-driven, constant-current stimulator (Phoresor, Iomed Inc., Salt Lake City, UT, USA) delivered $1.2 \mathrm{~mA}$ of electrical current to a participant's scalp by means of saline-dampened electrodes. For the anodal stimulation, the tDCS current ramped up over the first few seconds, and then remained on during the 20-min stimulation period. The sham-(control) tDCS was identical to the anodal stimulation, except that the experimenter reduced the current to zero after it ramped up for $30 \mathrm{~s}$; the current then stayed at zero for the remaining time period. Participants reported a tingly or itchy sensation at the start of the stimulation, which typically faded away after a few seconds. This sensation was present for both anodal- and sham-tDCS. Gandiga et al. (2006) found that naive participants were not able to distinguish between actual tDCS and sham-tDCS as we applied them in the present study. The application of tDCS began 5 min after the start of MIT, and continued for 5 min after the end of the MIT session. During the 5-min break after the end of MIT and before the end of the stimulation, the patients rested before completing the verbal fluency tasks.

\section{TASK}

Participants completed a battery of verbal fluency tests before and after each stimulation session. The tasks included the automatic production of verbal sequences (e.g., counting from 1 to 21 , reciting the days of the week, months of the year, and the United States' Pledge of Allegiance), describing flash card-like scenes, and picture naming. We chose the flash card-like scenes from drawings associated with the MIT intervention (Helm-Estabrooks and Albert, 1991). For the naming task we selected a set of colored pictures from the Snodgrass-Vanderwart inventory for naming, and a black and white line drawing of a scenario from the (BDAE 2nd Edn; Goodglass and Kaplan, 1983). Participants completed the automatic response items in the same order for each testing session. Pictures for the naming task were shown in a new random order for each session. We determined the number of items used in the automatic speech and picture naming tasks based on the ability of the participant, so that no testing session exceeded $30 \mathrm{~min}$. We limited the duration of the testing sessions in order to avoid excessive fatigue for the patients. Patients were instructed to simply try their best during each testing session, both pre- and post-therapy.

This was a randomized, cross-over, sham-controlled, doubleblind study in which the participants, the therapist, and the experimenter conducting and scoring the testing were all blind to stimulation condition.

\section{DATA ANALYSIS}

We calculated the dependent variable as the percentage of change ("proportional change") in the sum duration of fluency measures from before the first of three stimulation sessions to after the last of three stimulation sessions. This calculation produced two values for each participant: (1) (post-anodal series - preanodal series)/(pre-anodal series), (2) (post-sham series - presham series)/(pre-sham series). To ensure equality for all four time-points of interest (pre-anodal, post-anodal, pre-sham, postsham), the calculation of the dependent variable only included durations for fluency items, or portions thereof, that were intact at all of these time-points. For example, on the task of counting from 1 to 21 , if a participant was able to count to 21 at all time-points except one, during which he only made it to 18 , the duration for counting from 1 to 18 was used at all time-points. Similarly, we only used pictures that a participant was able to name at all four time-points of interest. Doing this ensured that the material at each time-point was identical in terms of content and what was actually spoken clearly. (Please see Table A1 in Appendix for details on individual participants' verbal output that met these criteria for inclusion in the analysis.) A rater who was blind to experimental condition measured the duration of the participants' fluency-test utterances using a waveform editor. The dependent variable was not sensitive to changes in the amount of verbal production, but to changes in the rate of verbal production, that is, how quickly the participant was able to utter appropriate, intelligible responses. The measure reflects fluency, with regard to ease of speech production. We compared the percentage of change for anodal- and sham-tDCS combined with MIT using a planned, two-tailed paired-samples $t$-test.

\section{RESULTS}

All six participants completed the experimental procedure. All patients tolerated the tDCS well and no adverse effects related to the application of tDCS were experienced. Averaged data for the effects of combining anodal and sham stimulation with MIT appear in Figure 2; and individual data for each participant are shown in Figure 3. We tested normality using the KolmogorovSmirnov test and found that the data were normal for both the sham $[Z(6)=0.48, p=0.98]$ and anodal $[Z(6)=0.49, p=0.97]$ conditions. The $t$-test comparing the effects of anodal-and shamtDCS yielded a significant result $[t(5)=3.22, p=0.02]$. We measured the effect size as Cohen's $d=1.98$. There was no difference

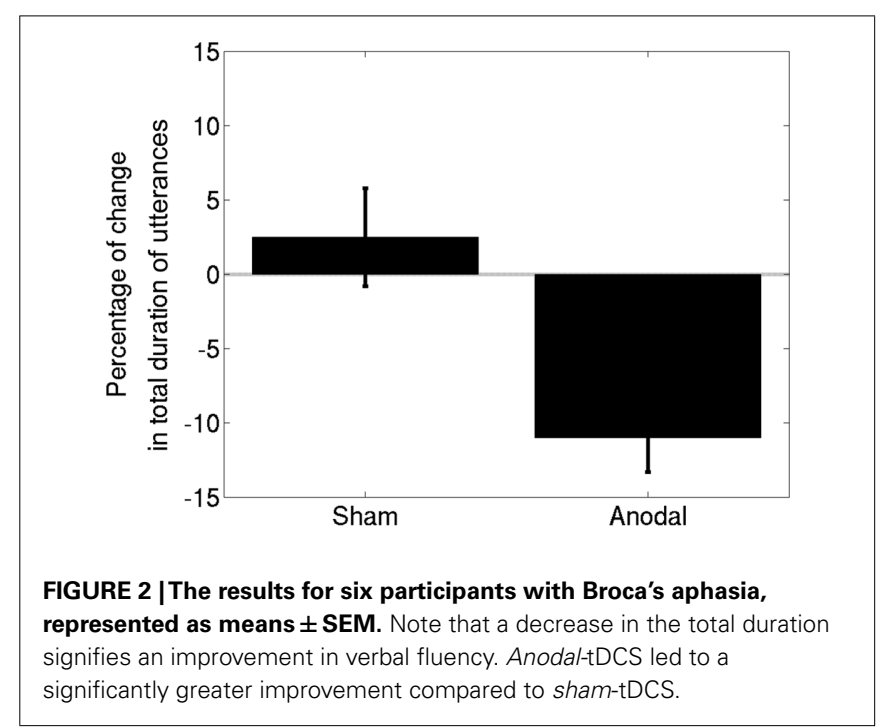




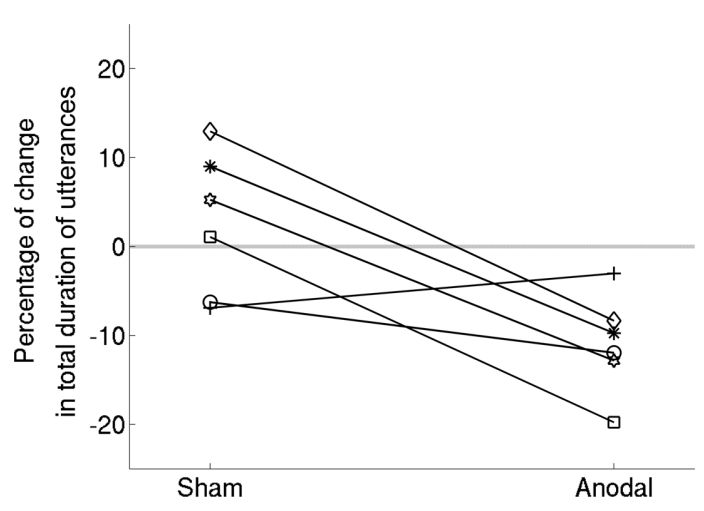

FIGURE 3 | Data shown separately for each participant. The trend for all but one participant is in the direction of greater improvement in the anodal-tDCS condition compared to sham.

between baseline performance in the anodal-tDCS condition and baseline performance in the sham condition. A paired-samples $t$-test comparing pre-anodal performance to pre-sham performance did not yield a significant result $[t(5)=-0.31, p=0.77]$. Thus, compared to sham-tDCS, applying anodal-tDCS to the right IFG during MIT produced a significantly greater improvement in verbal fluency while the verbal fluency in the sham-tDCS-MIT condition did not change significantly.

\section{DISCUSSION}

The results of this study provide evidence that applying "real" anodal-tDCS to the right IFG during MIT can augment the beneficial effects of the intonation-based speech therapy. By upregulating excitability in the right IFG, the anodal-tDCS may have increased synaptic plasticity in brain areas that are engaged by MIT and likely, drive the MIT-induced recovery process. We posit that increasing excitability in the right IFG with tDCS further engaged that area of the brain and thus, facilitated verbal output and fluency.

Future research will investigate whether the positive effects of tDCS were due to the particular placement of the anodal electrode over the right posterior IFG, or if anodal stimulation over other brain areas, such as the right anterior temporal cortex, could also improve upon the beneficial effects of MIT. It also remains unknown whether tDCS, as applied in this study, exerts a positive influence on language recovery only when combined with a behavioral speech therapy, or if tDCS could be effectively used on its own to improve verbal fluency for stroke patients such as those who participated in this study. The evidence from several recent cross-over studies suggests that beneficial effects are achieved only when real tDCS is simultaneously combined with behavioral therapy - neither behavioral nor speech therapy alone led to beneficial effects in these studies (Baker et al., 2010; Floel et al., 2011; Fridriksson et al., 2011), although one must consider the relatively few behavioral/speech therapy sessions that were done in these studies, as well as the total number of MIT sessions (3) in our current study. Nevertheless, the effects that were achieved by adding brain stimulation to behavioral interventions suggest that the combined approach might lead to much greater effects than a behavioral intervention alone. A previous review examining the intensity of the behavioral intervention in experimental aphasia studies (Bhogal et al., 2003) concluded that positive studies testing behavioral interventions had applied at least $60 \mathrm{~h}$ of treatment. This is many more hours than has been tested in the sham-control arm of experimental studies (Baker et al., 2010; Floel et al., 2011; Fridriksson et al., 2011; current study). Nevertheless, effects in the real-stimulation arm of recent experimental studies suggest that the addition of simultaneous brain stimulation to behavioral interventions might lead to stronger effects with fewer therapy sessions. Because the brain's plasticity that facilitates post-stroke language recovery may involve the development of latent neural connections in the undamaged brain, it is possible that modulating cortical excitability with noninvasive brain stimulation will have its greatest impact when a behavioral therapy takes advantage of the resulting neuro-plastic changes, as has been suggested in experimental animal studies (Fritsch et al., 2010).

While some aspects of other studies that also applied tDCS to facilitate recovery from non-fluent aphasia may have been similar to ours, a striking difference was that we stimulated a different area of the brain. Monti et al. (2008), for example, stimulated left fronto-temporal cortex, centered on Broca's area, and found an effect when applying $2 \mathrm{~mA}$ of cathodal stimulation over the left fronto-temporal electrode. Of particular interest here is that the anodal electrode in the Monti et al. (2008) design was placed over the right supraorbital region. Baker et al. (2010) and Fridriksson et al. (2011) both targeted peri-lesional areas in the left hemisphere with $1 \mathrm{~mA}$ and found increased accuracy on trained items in nonfluent patients (Baker et al., 2010; as well as an improvement in reaction time on trained items and a trend on untrained items in fluent patients; Fridriksson et al., 2011). We did not include a left hemisphere stimulation condition in the current study for three primary reasons. First, the patients in our study had little or no surviving brain tissue in the posterior IFG and surrounding brain structures of the left hemisphere (Figure 1; shows that at least four patients had lesions that involved the posterior IFG directly). For these patients, the best, and possibly only chance of recovery involved recruitment of brain regions in the right hemisphere to compensate for damaged language centers in the left hemisphere. Second, we chose to stimulate the right hemisphere because preliminary brain-imaging research provided evidence that MIT-induced improvements in speech output correlated with increased activity in the right IFG (Schlaug et al., 2008a). Our choice of location for stimulation was aimed at complementing the effects of MIT on brain activity. And third, stimulating over the damaged hemisphere of patients with relatively large lesions could generate an unpredictable current distribution and lead to correspondingly unpredictable effects on brain activity. This may explain some of the variability in results obtained from stimulating the left hemisphere in the treatment of non-fluent aphasia Fridriksson et al. (2011) and Baker et al. (2010) found that anodal stimulation led to speech improvements, whereas Monti et al. (2008) found that cathodal but not anodal stimulation produced improvements. Furthermore, a new study was just published in which anodal stimulation was applied to the right hemisphere 
and coupled with picture naming training (similar to Baker et al., 2010; Fridriksson et al., 2011), which led to a significant increase in accuracy on trained items (Floel et al., 2011).

The question of whether it would be best to apply tDCS before, during, or after a behavioral therapy also remains unanswered. If the effect of tDCS is greatest on performance, then applying the stimulation before or during therapy would be ideal for priming the brain in a specific area. However, if the beneficial effect of tDCS is on consolidation, then applying the stimulation either during or after therapy would have a greater effect. We chose to apply tDCS during MIT for the following reasons: (1) a number of tDCS studies have found effects on performance during stimulation (Antal et al., 2001; Nitsche et al., 2003b; Kincses et al., 2004; Rogalewski et al., 2004; Fregni et al., 2005; Hummel et al., 2005; Iyer et al., 2005; Boggio et al., 2006b; Hesse et al., 2007); (2) in the context of neurorehabilitation, applying stimulation during behavioral therapy is more efficient as it saves time for both the patient and the therapist/physician; (3) stimulation during the therapy has the potential to influence both the performance and the consolidation phases of learning; and (4) experiments in animals have shown that the combination of peripheral and central stimulation enhances synaptic plasticity more than central stimulation alone (Fritsch et al., 2010).

One primary limitation of this study was that we did not collect data to measure the longevity of any positive effect. As a result, we cannot comment on how long the effects of combined tDCS and MIT might last beyond the treatment period. For example, will the effects on performance continue for several days or weeks after the end of the combined therapy? It will be important to answer this question in order to determine whether and how often to use maintenance sessions to prolong potential benefits of the treatment. It also remains unknown how many tDCS + MIT sessions would be most effective. The current study used three, however, the benefits are likely to grow with additional sessions. Future studies will investigate the dose effects of combining tDCS with MIT to identify the optimal number of sessions.

Another limitation of this study concerns determining the exact site of stimulation. Based on previous research and our own pilot

\section{REFERENCES}

Adkins-Muir, D. L., and Jones, T. A. (2003). Cortical electrical stimulation combined with rehabilitative training: enhanced functional recovery and dendritic plasticity following focal cortical ischemia in rats. Neurol. Res. 25, 780-788.

Albert, M. L., Sparks, R. W., and Helm, N. A. (1973). Melodic intonation therapy for aphasia. Arch. Neurol. 29, 130-131.

Antal, A., Nitsche, M. A., Kruse, W., Kincses, T. Z., Hoffmann, K.-P., and Paulus, W. (2004). Direct current stimulation over V5 enhances visuomotor coordination by improving motion perception in humans. J. Cogn. Neurosci. 16, 521-527.

Antal, A., Nitsche, M. A., and Paulus, W. (2001). External modulation of visual perception in humans. $\mathrm{Neu}$ roreport 12, 3553-3555

Baker, J. M., Rorden, C., and Fridriksson, J. (2010). Using transcranial direct-current stimulation to treat stroke patients with aphasia. Stroke 41, 1229-1236.

Bhogal, S. K., Teasell, R., and Speechley, M. (2003). Intensity of aphasia therapy, impact on recovery. Stroke 34, 987-993.

Blasi, V., Young, A. C., Tansy, A. P., Petersen, S. E., Snyder, A. Z., and Corbetta, M. (2002). Word retrieval learning modulates right frontal cortex in patients with left frontal damage. Neuron 36, 159-170.

Boggio, P. S., Castro, L. O., Savagim, E. A., Braite, R., Cruz, V. C., Rocha, R. R., Rigonatti, S. P., Silva, M. T., and Fregni, F. (2006a). Enhancement of non-dominant hand motor function

study, we located the right posterior IFG as the right hemisphere homolog of Broca's area, using the 10-20 International EEG system for electrode placement. Considering the anatomical variability from one participant to the next, we may have stimulated slightly different brain areas in each of the six patients. The use of frameless stereotaxy in combination with MRI structural images for each participant would have enabled us to center the stimulation electrode with greater accuracy. However, due to the size of the electrode we used, it is highly likely that we did stimulate the area of interest even if there was some variability in its exact location across participants.

Given the positive nature of the results, this study shows that tDCS has the potential to augment the benefits of an intonationbased speech therapy. We found that three sessions of real tDCS + MIT led to a significant improvement in verbal fluency compared to an equivalent series of sham-tDCS + MIT. Although this is a preliminary finding with only six patients, the general implications extend beyond the domain of speech recovery, and into other areas of rehabilitation, such as motor recovery, cognitive training, and psychiatric treatment. Potentially, adding tDCS to any behavioral therapy may augment the positive effects. In the domain of speech therapy for severe non-fluent aphasia, this study provides evidence that anodal-tDCS + MIT applied to the right posterior IFG in non-fluent aphasic patients can facilitate improvements in linguistic fluency, and, presumably, in quality of life for stroke survivors.

\section{ACKNOWLEDGMENTS}

This work was supported by grants from the National Institute of Neurological Disorders and Stroke to Bradley W. Vines (NS053326) and to Gottfried Schlaug (RO1 DC008796). Gottfried Schlaug also acknowledges support from the Mary Crown and William Ellis Family Fund and the Rosalyn and Richard Slifka Family Fund. Bradley W. Vines also acknowledges support from the Grammy Foundation and the Michael Smith Foundation for Health Research. We thank Sarah Marchina for her help in generating Figure 1.

by anodal transcranial direct current stimulation. Neurosci. Lett. 404 232-236.

Boggio, P. S., Ferrucci, R., Rigonatti, S. P., Covre, P., Nitsche, M., Pascual-Leone, A., and Fregni, F. (2006b). Effects of transcranial direct current stimulation on working memory in patients with Parkinson's disease. J. Neurol. Sci. 249, 31-38.

Bonakdarpour, B., Eftekharzadeh, A., and Ashayeri, H. (2000). Preliminary report on the effects of melodic intonation therapy in the rehabilitation of Persian aphasic patients. Iran. J. Med. Sci. 25, 156-160.

Brown, S., Martinez, M. J., Hodges, D. A., Fox, P. T., and Parsons, L. M. (2004). The song system of the human brain. Brain Res. Cogn. Brain Res. 20, 363-375.
Cattaneo, Z., Pisoni, A., and Papagno, C. (2011). Transcranial direct current stimulation over Broca's region improves phonemic and semantic fluency in healthy individuals. Neuroscience 183, 64-70.

Celnik, P., Paik, N. J., Vandermeeren, Y., Dimyan, M., and Cohen, L. G. (2009). Effects of combined peripheral nerve stimulation and brain polarization on performance of a motor sequence task after chronic stroke. Stroke 40 , 1764-1771.

Fiori, V., Coccia, M., Marinelli, C. V., Vecchi, V., Bonifazi, S., Ceravolo, M. G., Provinciali, L., Tomaiuolo, F., and Marangolo, P. (2010). Transcranial direct current stimulation improves word retrieval in healthy and nonfluent aphasic subjects. J. Cogn. Neurosci. 23, 2309-2323. 
Floel, A., Meinzer, M., Kirstein, R., Nijhof, S., Deppe, M., Knecht, S., and Breitenstein, C. (2011). Shortterm anomia training and electrical brain stimulation. Stroke 42, 2065-2067.

Fregni, F., Boggio, P. S., Nitsche, M., Bermpohl, F., Antal, A., Feredoes, E., Marcolin, M. A., Rigonatti, S. P., Silva, M. T., Paulus, W., and PascualLeone, A. (2005). Anodal transcranial direct current stimulation of prefrontal cortex enhances working memory. Exp. Brain Res. 166, 23-30.

Fridriksson, J., Richardson, J. D., Baker, J. M., and Rorden, C. (2011). Transcranial direct current stimulation improves naming reaction time in fluent aphasia: a double-blind, sham-controlled study. Stroke 42, 819-821.

Fritsch, B., Reis, J., Martinowich, K., Schambra, H. M., Yuanyuan, J., Cohen, L. G., and Lu, B. (2010). Direct current stimulation promotes BDNF-dependent synaptic plasticity: potential implications for motor learning. Neuron 66, 198-204.

Gandiga, P. C., Hummel, F. C., and Cohen, L. G. (2006). Transcranial DC stimulation (tDCS): a tool for double-blind sham-controlled clinical studies in brain stimulation. Clin. Neurophysiol. 117, 845-850.

Gerstman, H. L. (1964). A case of aphasia. J. Speech Hear. Disord. 29, 89-91.

Geschwind, N. (1971). Current concepts: aphasia. N. Engl. J. Med. 284, 654-656.

Goldstein, K. (1942). After Effects of Brain-Injuries in War: Their Evaluation and Treatment. New York: Grune and Stratton.

Goodglass, H., and Geschwind, N. (1976). "Language disorders," in Handbook of Perception: Language and Speech, Vol. 7, eds E. Carterette and M. P. Friedman (New York: Academic Press), 390-428.

Goodglass, H., and Kaplan, E. (1983). Boston Diagnostic Aphasia Examination, 2nd Edn. Philadelphia: Lea and Febiger.

Hamilton, R. H., Chrysikou, E. G., and Coslett, B. (2011). Mechanisms of aphasia recovery after stroke and the role of noninvasive brain stimulation. Brain Lang. 118, 40-50.

Hebert, S., Racette, A., Gagnon, L., and Peretz, I. (2003). Revisiting the dissociation between singing and speaking in expressive aphasia. Brain 126, 1-13.

Heiss, W. D., and Thiel, A. (2006). A proposed regional hierarchy in recovery of post-stroke aphasia. Brain Lang. 98, 118-123.
Helm-Estabrooks, N., and Albert, M. L. (1991). Manual of Aphasia Therapy. Austin: Pro-Ed.

Hesse, S., Werner, C., Schonhardt, E. M., Bardeleben, A., Jenrich, W., and Kirker, S. G. (2007). Combined transcranial direct current stimulation and robot-assisted arm training in subacute stroke patients: a pilot study. Restor. Neurol. Neurosci. 25, 9-15.

Holland, A. L., Fromm, V., DeRuyter, F., and Stein, M. (1996). Treatment efficacy: aphasia. J. Speech Hear. Res. 39, S27-S36.

Homan, R. W., Herman, J., and Purdy, P. (1987). Cerebral location of international 10-20 system electrode placement. Electroencephalogr. Clin. Neurophysiol. 66, 376-382.

Hummel, F., Celnik, P., Giraux, P., Floel, A., Wu, W.-H., Gerloff, C., and Cohen, L. G. (2005). Effects of non-invasive cortical stimulation on skilled motor function in chronic stroke. Brain 128, 490-499.

Iyer, M. B., Mattu, U., Grafman, J., Lomarev, M., Sato, S., and Wassermann, E. M. (2005). Safety and cognitive effect of frontal DC brain polarization in healthy individuals. Neurology 64, 872-875.

Jeffries, K. J., Fritz, J. B., and Braun, A. R. (2003). Words in melody: an H-2 O-15 PET study of brain activation during singing and speaking. Neuroreport 14, 749-754.

Keith, R. L., and Aronson, A. E. (1975). Singing as therapy for apraxia of speech and aphasia: report of a case. Brain Lang. 2, 483-488.

Kertesz, A., and McCabe, P. (1977). Recovery patterns and prognosis in aphasia. Brain 100, 1-18.

Kertesz, A., Lesk, D., and McCabe, P. (1977). Isotope localization of infarcts in aphasia. Arch. Neurol. 34, 590-601.

Kincses, T. Z., Antal, A., Nitsche, M. A., Bartfai, O., and Paulus, W. (2004). Facilitation of probabilistic classification learning by transcranial direct current stimulation of the prefrontal cortex in the human. Neuropsychologia 42, 113-117.

Kinsella, G., Prior, M. R., and Murray, G. (1988). Singing ability after right and left sided brain damage. A research note. Cortex 24, 165-169.

Laughlin, S. A., Naeser, M. A., and Gordon, W. P. (1979). Effects of three syllable durations using the melodic intonation therapy technique. J. Speech Hear. Res. 22, 311-320.

Liebetanz, D., Nitsche, M. A., Tergau, F., and Paulus, W. (2002). Pharmacological approach to the mechanisms of transcranial DC- stimulation-induced after-effects of human motor cortex excitability. Brain 125, 2238-2247.

Lindenberg, R., Renga, V., Zhu, L. L., Nair, D., and Schlaug, G. (2010). Bihemispheric brain stimulation facilitates motor recovery in chronic stroke patients. Neurology 75, 2176-2184.

Marchina, S., Zhu, L. L., Norton, A., Zipse, L., Wan, C. Y., and Schlaug, G. (2011). Impairment of speech production predicted by lesion load of the arcute fasciculus. Stroke 42 , 2251-2256.

Mimura, M., Kato, M., Sano, Y., Kojima, T., Naeser, M., and Kashima, H. (1998). Prospective and retrospective studies of recovery in aphasia. Changes in cerebral blood flow and language functions. Brain 121, 2083-2094.

Mohr, J. P., Pessin, M. S., Finkelstein, S., Funkenstein, H. H., Duncan, G. W., and Davis, K. R. (1978). Broca aphasia: pathologic and clinical. Neurol ogy 28, 311-324

Monti, A., Cogiamanian, F., Marceglia, S., Ferrucci, R., and Mameli, F. (2008). Improved naming after transcranial direct current stimulation in aphasia. J. Neurol. Neurosurg. Psychiatry 79, 451-453.

Nair, D. N., Renga, V., Hamelin, S., Pascual-Leone, A., and Schlaug, G. (2008). Improving motor function in chronic stroke patients using simultaneous occupational therapy and tDCS. Stroke 39, 542.

Nitsche, M. A., Fricke, K., Henschke, U., Schlitterlau, A., Liebetanz, D., Lang, N., Henning, S., Tergau, F., and Paulus, W. (2003a). Pharmacological modulation of cortical excitability shifts induced by transcranial DC stimulation. J. Physiol. 553, 293-301.

Nitsche, M. A., Schauenburg, A., Lang, N., Liebetanz, D., and Exner, C. (2003b). Facilitation of implicit motor learning by weak transcranial direct current stimulation of the primary motor cortex in the human. $J$. Cogn. Neurosci. 15, 619-626.

Nitsche, M. A., and Paulus, W. (2000). Excitability changes induced in the human motor cortex by weak transcranial direct current stimulation. $J$. Physiol. 527, 633-639.

Okamoto, M., Dan, H., Sakamoto, K., Takeo, K., Shimizu, K., Kohno, S., Oda, I., Isobe, S., Suzuki, T., Kohyama, K., and Dan, I. (2004). Three-dimensional probabilistic anatomical cranio-cerebral correlation via the international 10-20 system oriented for transcranial functional brain mapping. $\mathrm{Neu}$ roimage 21, 99-111.
Ozdemir, E., Norton, A., and Schlaug, G. (2006). Shared and distinct neural correlates of singing and speaking. Neuroimage 33, 628-635.

Pedersen, P. M., Vinter, K., and Olsen, T. S. (2004). Aphasia after stroke: type, severity and prognosis. The Copenhagen aphasia study. Cerebrovasc. Dis. 17, 35-43

Pizzamiglio, L., Galati, G., and Committeri, G. (2001). The contribution of functional neuroimaging to recovery after brain damage: a review. Cortex 37, 11-31.

Priori, A., Berardelli, A., Rona, S., Accornero, N., and Manfredi, M. (1998). Polarization of the human motor cortex through the scalp. Neuroreport 9, 2257-2260.

Racette, A., Bard, C., and Peretz, I. (2006). Making non-fluent aphasics speak: sing along! Brain 129, 2571-2584.

Riecker, A., Ackermann, H., Wildgruber, D., Dogil, G., and Grodd, W. (2000). Opposite hemispheric lateralization effects during speaking and singing at motor cortex, insula and cerebellum. Neuroreport 11 , 1997-2000.

Robey, R. R. (1994). The efficacy of treatment for aphasic persons: a meta-analysis. Brain Lang. 47, 582-608.

Rogalewski, A., Breitenstein, C., Nitsche, M. A., Paulus, W., and Knecht, S. (2004). Transcranial direct current stimulation disrupts tactile perception. Eur. J. Neurosci. 20, 313-316.

Rosen, H. J., Petersen, S. E., Linenweber, M. R., Snyder, A. Z., White, D. A., Chapman, L., Dromerick, A W., Fiez, J. A., and Corbetta, M. D. (2000). Neural correlates of recovery from aphasia after damage to left inferior frontal cortex. Neurology 55, 1883-1894.

Saur, D., Lange, R., Baumgaertner, A., Schraknepper, V., Willmes, K., Rijntjes, M., and Weiller, C. (2006). Dynamics of language reorganization after stroke. Brain 129, 13711384.

Schlaug, G., Marchina, S., and Norton, A. (2008a). From singing to speaking: why singing may lead to recovery of expressive language function in patients with Broca's aphasia. Music Percept. 25, 315-323.

Schlaug, G., Norton, A., and Marchina, S. (2008b). The role of the right hemisphere in post-stroke language recovery. Stroke 39, 542-543.

Schlaug, G., Renga, V., and Nair, D. (2008c). Transcranial direct current stimulation in stroke recovery. Arch. Neurol. 65, 1571-1576. 
Schlaug, G., Marchina, S., and Norton, A. (2009). Evidence for plasticity in white-matter tracts of patients with chronic Broca's aphasia undergoing intense intonation-based speech therapy. Ann. N. Y. Acad. Sci. 1169, 385-394.

Schlaug, G., Marchina, S., and Wan, C. Y. (2011). The use of noninvasive brain stimulation techniques to facilitate recovery from post-stroke aphasia. Neuropsychol. Rev. 21, 288-301.

Schlaug, G., Norton, A., Marchina, S., Zipse, L., and Wan, C. Y. (2010). From singing to speaking: facilitating recovery from nonfluent aphasia. Future Neurol. 5, 657-665.

Schlaug, G., and Renga, V. (2008). Transcranial direct current stimulation - a non-invasive tool to facilitate stroke recovery. Expert Rev. Med. Devices 5, 759-768.

Schuppert, M., Münte, T., Wieringa, B., and Altenmuller, E. (2000). Receptive amusia: evidence for cross-hemispheric neural networks underlying music processing strategies. Brain 123, 546-559.

Sparing, R., Meister, I. G., Wienemann, M., Buelte, D., Staedtgen, M., and Boroojerdi, B. (2007). Taskdependent modulation of functional connectivity between hand motor cortices and neuronal networks underlying language and music: a transcranial magnetic stimulation study in humans. Eur. J. Neurosci. 25, 319-323.

Sparks, R., Helm, N., and Albert, M. (1974). Aphasia rehabilitation resulting from melodic intonation therapy. Cortex 10, 303-316.

Stefan, K., Kunesch, E., Cohen, L. G., Benecke, R., and Classen, J. (2000). Induction of plasticity in the human motor cortex by paired associative stimulation. Brain 123, 572-584.

Thiel, A., Herholz, K., Koyuncu, A., Ghaemi, M., Kracht, L. W., Habedank, B., and Heiss, W. D. (2001). Plasticity of language networks in patients with brain tumors: a positron emission tomography activation study. Ann. Neurol. 50, 620-629.

Vines, B. W., Cerruti, C., and Schlaug, G. (2008a). Dual-hemisphere tDCS facilitates greater improvements in motor performance compared to uni-hemisphere stimulation. $B M C$ Neurosci. 9, 103. doi: 10.1186/14712202-9-103

Vines, B. W., Nair, D. G., and Schlaug, G. (2008b). Modulating activity in the motor cortex affects performance for the two hands differently depending upon which hemisphere is stimulated. Eur. J. Neurosci. 28, 1667-1673.

Vines, B. W., Nair, D. G., and Schlaug, G. (2006a). Contralateral and ipsilateral motor effects after transcranial direct current stimulation. Neuroreport 17, 671-674.

Vines, B. W., Schnider, N., and Schlaug, G. (2006b). Testing for causality with tDCS: pitch memory and the left supramarginal gyrus. Neuroreport 17, 1047-1050.

Wan, C., Rüber, T., Hohmann, A., and Schlaug, G. (2010). The therapeutic effects of singing in neurological disorders. Music Percept. 27, 287-295.

Wilson, S. J., Parsons, K., and Reutens, D. C. (2006). Preserved singing in aphasia: a case study of the efficacy of melodic intonation therapy. Music Percept. 24, 23-35.

Winhuisen, L., Thiel, A., Schumacher, B., Kessler, J., Rudolf, J., Haupt, W. F., and Heiss, W. D. (2005). Role of the contralateral inferior frontal gyrus in recovery of language function in post stroke aphasia: a combined repetitive transcranial magnetic stimulation and positron emission tomography study. Stroke 36, 1759-1763.
Zheng, X., Alsop, D. C., and Schlaug, G. (2011). Effects of transcranial direct current stimulation (tDCS) on human regional cerebral blood flow. Neuroimage 58, 26-33.

Conflict of Interest Statement: The authors declare that the research was conducted in the absence of any commercial or financial relationships that could be construed as a potential conflict of interest.

Received: 02 April 2011; accepted: 26 August 2011; published online: 26 September 2011.

Citation: Vines BW, Norton $A C$ and Schlaug $G$ (2011) Non-invasive brain stimulation enhances the effects of melodic intonation therapy. Front. Psychology 2:230. doi: 10.3389/fpsyg.2011.00230

This article was submitted to Frontiers in Auditory Cognitive Neuroscience, a specialty of Frontiers in Psychology. Copyright (c) 2011 Vines, Norton and Schlaug. This is an open-access article subject to a non-exclusive license between the authors and Frontiers Media $S A$, which permits use, distribution and reproduction in other forums, provided the original authors and source are credited and other Frontiers conditions are complied with. 


\section{APPENDIX}

Table A1 | For each participant, this table includes spoken material that was intact at all four time-points, and thus, was used in the analysis.

Task category Specific task Intact portion(s)

\section{PARTICIPANT 1}

Automatic speech

Picture description

\section{PARTICIPANT 2}

Automatic speech

Pledge of allegiance

Counting

Days of the week

Months of the year

Picture description

Describe these scenes

Picture naming

Name the object pictured

\section{ntact portion(s)}

"I pledge allegiance to the flag of the United States of America"

$1-15$

Thursday through Sunday

March through May

"Cup of tea," "cream and sugar," "call the doctor," ". . . are my shoes," "brush your teeth," "clean it up" "my name is what's your name?" "turn off the light,"

Abacus, acorn, apple, bathroom, beaver, cactus, giraffe, helicopter, money, pelican, pencil, pyramid, scissors, unicorn, water, whistle, window, zebra

"I pledge allegiance to the flag of the United States of America, and to the Republic for which it stands, one nation under God, indivisible, with liberty and justice for all."

$1-10$

Monday through Sunday

January through October

"Open up," "go to bed," "call the doctor," "close the window," "where are my shoes?" "brush your teeth," "wait for me," "hi, my name is ___," "cup of coffee," "cream and sugar," "turn off the light," "I don't understand," "pass the butter"

Abacus, accordion, acorn, asparagus, beaver, cactus, camel celery compass, dominoes hammock, hanger, harmonica, igloo, mushroom, octopus, overalls, palette, pelican, piano, pretzel, racket, rhinoceros, seahorse, strawberry, toothbrush, tripod, unicorn, volcano, wheelchair

\section{PARTICIPANT 3}

Automatic speech

Pledge of allegiance

Counting

Days of the week

Months of the year

Picture description

Describe these scenes

$-$

1-8

Monday through Tuesday

March through June

"Butter," "call the doctor, please," "shut the window, please," "brush your teeth," "where are my shoes?" "come over here," "my name is ___, " . . .you doing?" "coffee," ". . . and cream," "turn off the light," "I don't understand"

Picture naming Name the object pictured pencil, piano, scissors, strawberry, toaster, whistle, zebra

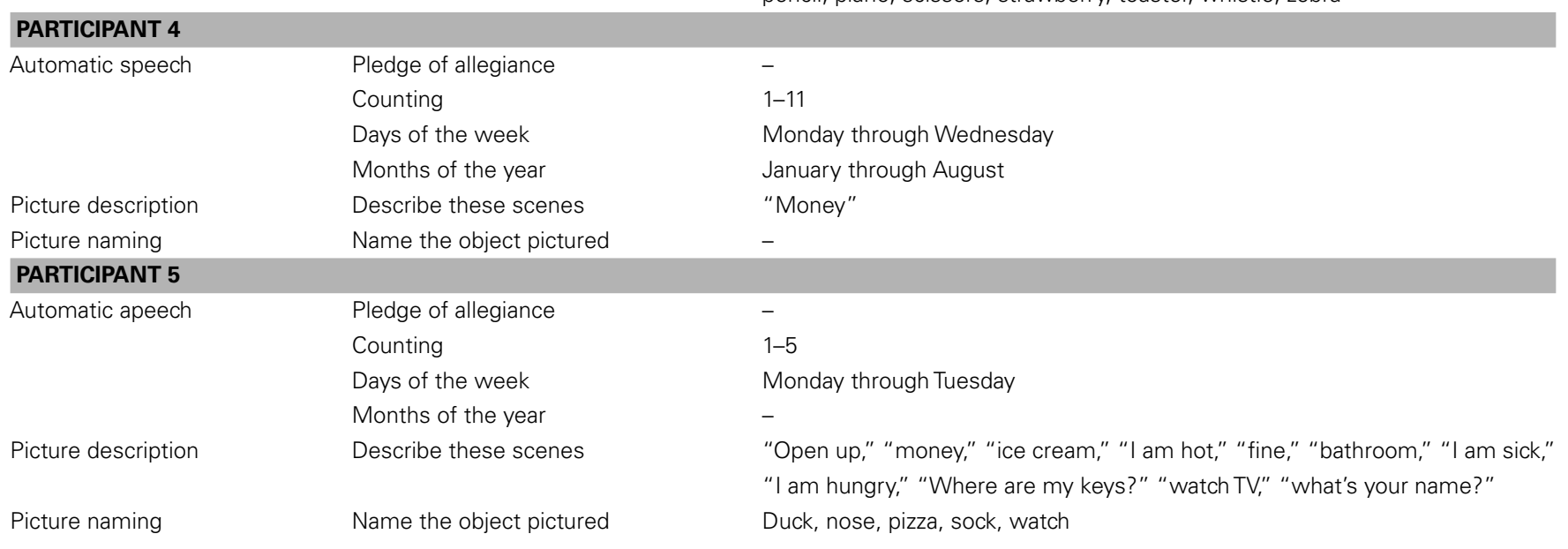

(Continued) 
Table A1 | Continued

\begin{tabular}{|c|c|c|}
\hline Task category & Specific task & Intact portion(s) \\
\hline \multicolumn{3}{|l|}{ PARTICIPANT 6} \\
\hline \multirow[t]{4}{*}{ Automatic speech } & Pledge of allegiance & $\begin{array}{l}\text { "...pledge allegiance to the flag of the United States of America, and to the } \\
\text { Republic for which it stands, one nation under God, indivisible..." }\end{array}$ \\
\hline & Counting & $1-20$ \\
\hline & Days of the week & Monday through Sunday \\
\hline & Months of the year & February through December \\
\hline Picture description & Describe these scenes & - \\
\hline Picture naming & Name the object pictured & - \\
\hline
\end{tabular}

